Bundesgesundheitsbl 2013 · 56:723-732

DOI 10.1007/s00103-013-1684-7

Online publiziert: 27. Mai 2013

c) Springer-Verlag Berlin Heidelberg 2013

\section{Additional material online}

An English full-text version of this article is available at SpringerLink under supplementary material: dx.doi.org/10.1007/s00103-013-1684-7

\section{Hintergrund und Fragestellung}

Im höheren Lebensalter spielt der Erhalt der körperlichen und geistigen Funktionsfähigkeit neben den vorhandenen chronischen und akuten Erkrankungen eine zunehmend wichtigere Rolle, um ein selbstständiges Leben zu führen. Funktionseinschränkungen bewirken eine Beeinträchtigung in der Durchführung von Alltagaktivitäten [1] und stehen in Zusammenhang mit einer erhöhten Sterblichkeit [2, 3]. Altersassoziierten Funktionseinschränkungen liegen vielfältige Ursachen zugrunde. Diese können akute Krankheitsereignisse wie ein Schlaganfall sein, chronische Erkrankungen wie Demenz, Arthrose oder rheumatoide Arthritis, mangelnde körperliche Aktivität oder Übergewicht [4], aber auch eine über das physiologische altersbedingte $\mathrm{Maß}$ hinausgehende $\mathrm{Ab}$ nahme der Muskelmasse und Muskelkraft (Sarkopenie) $[5,6]$.

Durch Messung der alltagsrelevanten Funktionsfähigkeit lassen sich Einschränkungen in objektiver Weise sichtbar machen und Folgen wie Behinderung im Alltag, Verlust von Autonomie und Lebensqualität abschätzen. In der Versorgung und Rehabilitation älterer Menschen spielt die systematische objektive Erfassung von Funktions- und Fähigkeitsstörungen (geriatrisches As-

J. Fuchs · M.A. Busch • A. Gößwald • H. Hölling • R. Kuhnert • C. Scheidt-Nave

Abteilung für Epidemiologie und Gesundheitsmonitoring, Robert Koch-Institut, Berlin

\title{
Körperliche und geistige Funktionsfähigkeit bei Personen im Alter von 65 bis 79 Jahren in Deutschland
}

\section{Ergebnisse der Studie zur Gesundheit Erwachsener in Deutschland (DEGS1)}

sessment) daher bereits eine bedeutende Rolle [7].

Bevölkerungsrepräsentative Daten zu Funktionsfähigkeit und -einschränkungen der Personen ab 65 Jahre in Deutschland sind bisher auf einzelne Funktionstests beschränkt [8]. Ein einheitlich verwendeter Standard zur Erfassung der Funktionsfähigkeit im Alter liegt derzeit nicht vor.

Valide bevölkerungsrepräsentative Daten zu Funktionsfähigkeiten sind aus mehreren Gründen wesentlich. Erstens tragen solche Daten dazu bei, die $\mathrm{Zu}$ sammenhänge zwischen Funktionseinschränkungen und medizinischen, aber auch soziodemografischen und umweltassoziierten Faktoren besser zu verstehen. Zweitens sind solche Daten unerlässlich, um Gesundheitszustand, Versorgungsbedarf und Präventionspotenzial in einer immer älter werdenden Gesellschaft unter Berücksichtigung verschiedener Dimensionen von Gesundheit (Krankheiten, Funktionseinschränkungen, Teilhabestörungen) $[9,10,11]$ einzuschätzen. Dies entspricht dem Konzept der Internationalen Klassifikation der Funktionsfähigkeit, Behinderung und Gesundheit der WHO [12].

Im Rahmen von DEGS1 wurde vor diesem Hintergrund erstmals auch eine Batterie etablierter Testverfahren zur objektiven Erfassung von alltagsrelevanten
Funktionsfähigkeiten in einen bundesweiten Gesundheitssurvey bei Erwachsenen integriert. Ziel des vorliegenden Beitrages ist es, die methodischen Standards zu beschreiben und erste Ergebnisse zur Verteilung von Funktionsfähigkeiten bei Personen im Alter von 65 bis 79 Jahren nach Alter, Geschlecht und Sozialstatus zu berichten.

\section{Methoden}

\section{Studiendesign und Stichprobe}

Die „Studie zur Gesundheit Erwachsener in Deutschland“ (DEGS) ist Bestandteil des Gesundheitsmonitorings des Robert Koch-Instituts (RKI). Konzept und Design von DEGS sind an anderer Stelle ausführlich beschrieben $[13,14,15,16$, 17]. Die erste Erhebungswelle (DEGS1) wurde von 2008 bis 2011 durchgeführt und umfasste Befragungen, Untersuchungen und Tests $[18,19]$. Zielpopulation war die in Deutschland lebende Bevölkerung im Alter von 18 bis 79 Jahren. DEGS1 hat ein Mischdesign, das gleichzeitig quer- und längsschnittliche Analysen ermöglicht. Hierbei wurde eine Einwohnermeldeamtsstichprobe gezogen, die ehemalige Teilnehmerinnen und Teilnehmer des Bundes-Gesundheitssurveys 1998 (BGS98) ergänzt. Insgesamt nahmen 8152 Personen teil, darunter 


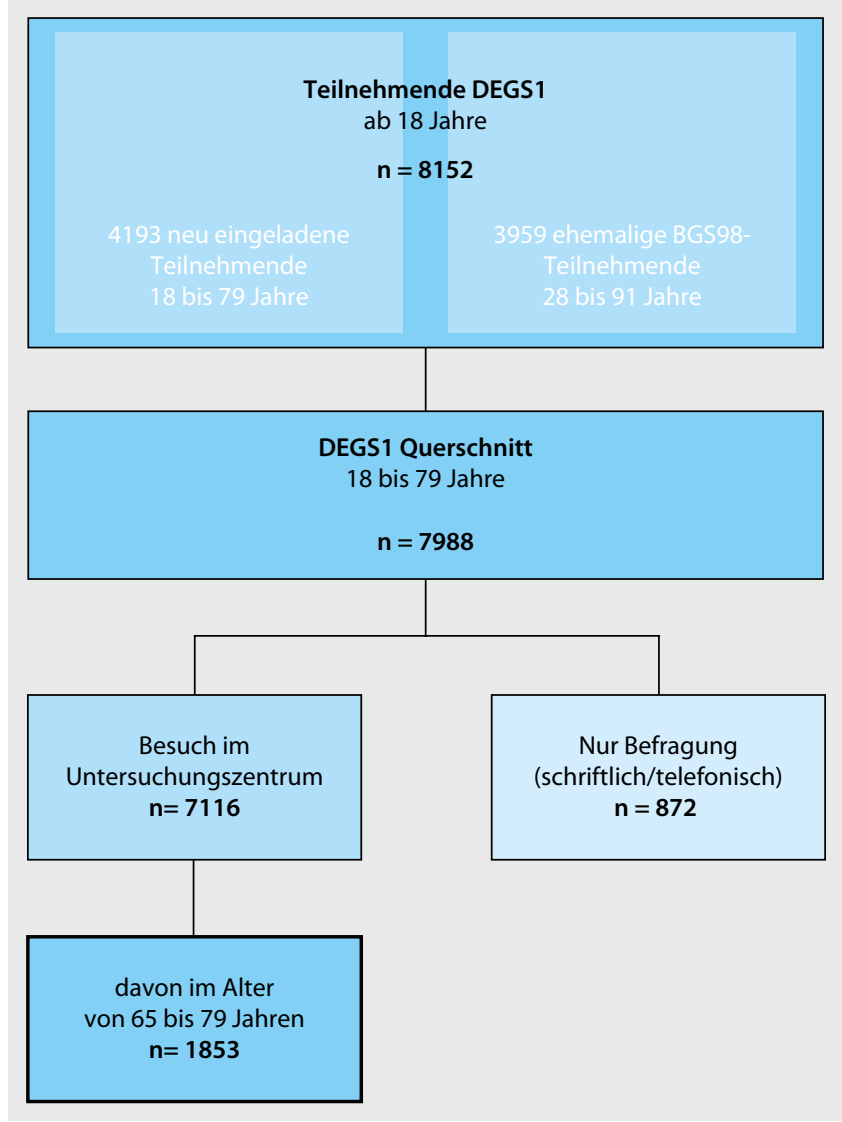

Abb. $1<$ Überblick über die Zahl der Teilnehmenden

4193 Ersteingeladene (Response 42\%) und 3959 ehemalige Teilnehmerinnen und Teilnehmer des BGS98 (Response 62\%). 7238 Personen besuchten eines der 180 Untersuchungszentren, 914 wurden ausschließlich befragt. Die Nettostichprobe [14] ermöglicht für den Altersbereich von 18 bis 79 Jahren repräsentative Querschnittanalysen und Trendaussagen im Vergleich mit dem BGS98 ( $\mathrm{n}=7988$, davon 7116 in Untersuchungszentren). Die Daten der erneut Teilnehmenden sind für Längsschnittanalysen nutzbar. Die hier vorgestellte Auswertung bezieht sich auf 1853 Personen im Alter von 65 bis 79 Jahren, die an den Untersuchungen im Untersuchungszentrum teilgenommen haben (• Abb. 1). Die im Folgenden vorgestellten Funktionstests wurden nur bei Personen im Alter ab 65 Jahre durchgeführt.

\section{Funktionstests}

Zur Erfassung der Funktionseinschränkungen wurden folgende international etablierte Funktionstests eingesetzt, die einfach durchzuführen sind, gut reproduzierbare Ergebnisse liefern und valide Messergebnisse mit Bedeutung für Behinderung im Alltag und Unterstützungsbedarf und Mortalität sowie Erfassung eines erhöhten Sturzrisikos liefern:

- Timed-up and Go-Test (TUG) [20, 21],

- Chair-Rise-Test [22],

- Balance-Testbatterie [22, 23],

- Messung der isometrischen ${ }^{1}$ Handgriffstärke [8, 24, 25],

- Zahlen-Symbol-Test [26, 27].

Für alle 5 der in DEGS1 eingesetzten Einzeltests ist eine prognostische Relevanz in längsschnittlichen Studien belegt [1, 28, 29].

Die Tests wurden von Studienassistentinnen durchgeführt, die vor Beginn der Studie umfangreich geschult und anschließend zertifiziert wurden, um die Durchführung nach standardisier-

\footnotetext{
1 Muskelkontraktion bei haltender oder statischer Tätigkeit mit Erhöhung der Muskelspannung ohne Änderung der Muskellänge.
}

ten Protokollen sicherzustellen. Während der Feldphase erfolgten regelmäßige Qualitätssicherungen und bei Bedarf Nachschulungen (s. auch [30] in diesem Heft).

\section{Timed-up and Go-Test (TUG)}

Mit dem Timed-up and Go-Test (TUG) liegt ein Test zur Erfassung von alltagsrelevanten Mobilitätseinschränkungen vor [20]. Gemessen wird die Zeit in Sekunden, die benötigt wird, um von einem Stuhl aufzustehen, in normaler Geschwindigkeit eine Strecke von $3 \mathrm{~m} \mathrm{zu}$ gehen, zu wenden, zurück zum Stuhl zu gehen, sich dort zu drehen und wieder hinzusetzen. Voraussetzung für die Durchführung ist die Gehfähigkeit. Beim Aufstehen dürfen die Armlehnen zum Abstützen benutzt werden, Gehhilfen wie Rollator, Gehstützen oder Stöcke dürfen bei Bedarf eingesetzt werden. Ausschlussgründe liegen vor, wenn die Gehfähigkeit auch mit Hilfsmitteln nicht vorhanden ist oder die Person blind oder stark sehbehindert ist. Ergebnisse quer- und längsschnittlicher Studien belegen die funktionale und prognostische Relevanz einer verringerten Gehgeschwindigkeit für Einschränkungen bei der Durchführung von Alltagsaktivitäten und Unterstützungsbedarf $[1,20,21]$ sowie ein erhöhtes Sturzrisiko [31]. Prognostisch relevante Schwellenwerte variieren in Abhängigkeit von der untersuchten Studienpopulation und der betrachteten Zielgröße. Ab einer benötigten Zeit von $20 \mathrm{~s}$ wird vom Vorliegen einer alltagsrelevanten Mobilitätsstörung ausgegangen [21].

\section{Chair-Rise-Test}

Der Chair-Rise-Test erfasst die Fähigkeit, sich von einer sitzenden in eine stehende Position zu begeben. Dies erfordert Kraft in den Beinen und ist eine Grundvoraussetzung für die Bewegungsfähigkeit und damit einer unabhängigen Lebensführung. Gemessen wird die Zeit in Sekunden, die benötigt wird, um fünfmal hintereinander von einem Stuhl aufzustehen und sich wieder hinzusetzen. Zur Durchführung des Tests sitzt die Person mit vor der Brust gekreuzten Armen aufrecht auf einem Stuhl ohne Armlehne mit $43 \mathrm{~cm}$ Sitzhöhe, ohne sich anzulehnen. Beide 
Füße stehen stabil etwa hüftbreit auseinander auf dem Boden. Die Person steht nach einem Probedurchgang (einmaliges Aufstehen) fünfmal ohne Zuhilfenahme der Arme auf, sodass sie aufrecht steht und Hüften und Knie gestreckt sind; dabei soll sie sowohl auf Schnelligkeit als auch auf Genauigkeit der Untersuchungsdurchführung achten. Am Ende des fünften Mals bleibt die Person stehen. Während der Durchführung des Tests wird sie nicht unterstützt oder ermutigt. Der Test wird nicht durchgeführt wenn ein eigenständiges Aufstehen nicht möglich ist, sich die Testdurchführung im Probedurchgang als unmöglich herausstellt oder die Stehfähigkeit nicht vorhanden oder eingeschränkt ist (z. B. bei Lähmungen, Amputationen oder Gleichgewichtsstörungen). Ein Zusammenhang zwischen niedrigeren Messwerten und einem erhöhten Risiko für Einschränkungen der Alltagsfähigkeit und Unterstützungsbedarf ist in längsschnittlichen epidemiologischen Studien wiederholt berichtet worden $[32,33,34]$.

\section{Balance-Tests}

Die Balance-Tests erfolgten gemäß der Untersuchungsdurchführung der hierarchischen Testbatterie der Short Physical Performance Battery (SPPB), die in internationalen epidemiologischen Studien eingesetzt wird [22, 35]. Sie wurde in DEGS1 um den Einbeinstand ergänzt, um den Schwierigkeitsgrad zu erhöhen und eine bessere Differenzierung zu erlangen [36]. Erfasst wird die Fähigkeit in 4 im Schwierigkeitsgrad ansteigenden Stufen, 10 s lang die jeweilige Position zu halten:

1. Romberg-Position: Füße stehen parallel dicht nebeneinander.

2. Semi-Tandem-Stand: Aus der Position mit parallel stehenden Füßen wird ein Fuß nach vorne gesetzt; dabei bestimmt die Person selbst, welcher Fuß vorgesetzt wird. Die Füße stehen um eine Fußbreite versetzt so, dass die Ferse des vorgesetzten Fußes auf einer Höhe mit der Fußspitze des anderen Fußes steht. Die Füße berühren sich jedoch nicht.

3. Tandem-Stand: Füße stehen in einer Linie hintereinander, die Ferse des vorangesetzten Fußes berührt die Spitze

Bundesgesundheitsbl 2013 · 56:723-732 DOI 10.1007/s00103-013-1684-7

(c) Springer-Verlag Berlin Heidelberg 2013

J. Fuchs · M.A. Busch · A. Gößwald · H. Hölling · R. Kuhnert · C. Scheidt-Nave

Körperliche und geistige Funktionsfähigkeit bei Personen

im Alter von 65 bis 79 Jahren in Deutschland. Ergebnisse der Studie zur Gesundheit Erwachsener in Deutschland (DEGS1)

\section{Zusammenfassung}

Die Funktionsfähigkeit spielt im Alter eine wichtige Rolle für ein selbstständiges Leben. In der Studie zur Gesundheit Erwachsener in Deutschland (DEGS1) wurden daher Timed-up and Go-Test (TUG), Chair-RiseTest, Balance-Tests, Greifkraft-Test und Zahlen-Symbol-Test (ZST) eingesetzt, um die körperliche und kognitive Funktionsfähigkeit von 65- bis 79-Jährigen in Deutschland bevölkerungsrepräsentativ zu beschreiben. Von den 1853 Personen zwischen 65 und 79 Jahren, die ins Untersuchungszentrum kamen, nahmen über $90 \%$ an den Funktionstests teil. Für den TUG wurden im Mittel 10,7 s benötigt, für den Chair-Rise-Test 11,8 s. Von den möglichen 5 Punkten im Balance-Test (nach FICSIT4-Protokoll) wurden im Mittel 3,9 Punkte erreicht. Die mittlere maximale Greifkraft lag bei 32,3 kg. Im ZST wurden 43,8 Zeichen richtig zugeordnet. In allen Fähigkeitsbereichen wurde eine Leistungsabnahme mit zunehmendem Alter deutlich; geschlechtsspezifische Unterschiede zeigen sich beim ChairRise-Test, Greifkraft-Test und ZST. Die objektive Erfassung körperlicher und kognitiver Funktionseinschränkungen in DEGS1 trägt zur Charakterisierung des Gesundheitszustandes Älterer bei und ist relevant für die Prävention und Gesundheitsförderung im höheren Lebensalter.

\section{Schlüsselwörter}

Funktionstests · Funktionsfähigkeit . Funktionseinschränkungen · Ältere · Gesundheitssurvey

\section{Physical and cognitive capabilities among persons aged 65-79 years in Germany. Results of the German Health Interview and Examination Survey for Adults (DEGS1)}

\section{Abstract}

In older age, physical and cognitive capabilities play an important role for independent living. For this reason, the German Health Interview and Examination Survey for Adults (DEGS1) included the Timed Up and Go test (TUG) and a chair-rise test, balance tests, a measurement of hand grip strength and the Digit Symbol Substitution Test (DSST) in order to representatively describe physical and cognitive performance of older people in Germany. Among 1,853 persons $65-79$ years of age who came to the study centre more than $90 \%$ participated in the performance tests. The average time needed to complete the TUG and chair-rise tests were 10.7 and $11.8 \mathrm{~s}$, respectively. On average, participants reached 3.9 of a maximum of 5 points in the balance tests (FICSIT4 protocol). Mean max- imum grip strength was $32.3 \mathrm{~kg}$. The mean number of correctly assigned symbols in the DSST was 43.8. In all functional capacity areas tested, performance declined with increasing age. There were differences by sex in the chair-rise test, hand grip strength and DSST. The objective measurement of physical and cognitive capabilities in DEGS1 contributes to describe the health status of older people with implications for health promotion and prevention. An English full-text version of this article is available at SpringerLink as supplemental.

\section{Keywords}

Physical performance $\cdot$ Physical and cognitive capabilities · Limitations · Aging · Health survey des anderen Fußes. Ein Abstand von maximal $1 \mathrm{~cm}$ wird toleriert, eine seitliche Abweichung sollte $2 \mathrm{~cm}$ nicht überschreiten.

4. Einbeinstand: Stehen auf einem Bein, jeweils rechts und links.
Die Tests werden in der Mitte des Raumes durchgeführt. Die Zeit, die die Person in der jeweiligen Position verharren kann, ohne die Füße zu verstellen, wird mit einer Stoppuhr gemessen. Nach $10 \mathrm{~s}$ wird der Test beendet. Ausschlussgründe liegen vor, wenn kein hüftbreites sicheres Stehen möglich ist, z. B. bei Beinamputa- 
Tab. 1 Charakteristika der Stichprobe für die Funktionstests (65 bis 79 Jahre) ( $n=1853)$

\begin{tabular}{|c|c|c|c|c|c|}
\hline & & \multicolumn{3}{|l|}{ Altersgruppe } & \multirow[t]{2}{*}{ Insgesamt } \\
\hline & & 65 bis 69 Jahre & 70 bis 74 Jahre & 75 bis 79 Jahre & \\
\hline \multirow[t]{3}{*}{ Frauen } & Gesamt & $33,6 \%$ & $42,4 \%$ & $24,0 \%$ & $53,9 \%$ \\
\hline & $95 \%-\mathrm{KI}$ & $30,2-37,7 \%$ & $38,5-46,4 \%$ & $20,5-27,7 \%$ & $51,5-56,4 \%$ \\
\hline & $\mathrm{n}_{\text {ungewichtet }}$ & 373 & 371 & 185 & 929 \\
\hline \multirow{9}{*}{$\begin{array}{l}\text { Sozial- } \\
\text { status }^{\text {a }}\end{array}$} & Niedrig & $22,0 \%$ & $29,5 \%$ & $36,9 \%$ & $28,7 \%$ \\
\hline & $95 \%-K I$ & $16,8-28,3 \%$ & $23,4-36,4 \%$ & $28,6-46,0 \%$ & $24,5-33,2 \%$ \\
\hline & $\mathrm{n}_{\text {ungewichtet }}$ & 64 & 81 & 56 & 201 \\
\hline & Mittel & $65,7 \%$ & $61,8 \%$ & $57,8 \%$ & $62,2 \%$ \\
\hline & $95 \%-\mathrm{Kl}$ & $59,3-71,5 \%$ & $55,2-68,0 \%$ & $48,7-66,4 \%$ & $57,8-66,4 \%$ \\
\hline & $\mathrm{n}_{\text {ungewichtet }}$ & 247 & 231 & 110 & 588 \\
\hline & Hoch & $12,3 \%$ & $8,7 \%$ & $5,3 \%$ & $9,1 \%$ \\
\hline & $95 \%-\mathrm{Kl}$ & $8,8-17,1 \%$ & $6,4-11,8 \%$ & $2,9-9,6 \%$ & $7,2-11,6 \%$ \\
\hline & $\mathrm{n}_{\text {ungewichtet }}$ & 61 & 54 & 17 & 132 \\
\hline \multirow[t]{3}{*}{ Männer } & Gesamt & $36,2 \%$ & $43,3 \%$ & $20,5 \%$ & $46,1 \%$ \\
\hline & $95 \%-\mathrm{Kl}$ & $32,6-40,0 \%$ & $39,4-47,3 \%$ & $17,4-23,9 \%$ & $43,6-48,5 \%$ \\
\hline & $\mathrm{n}_{\text {ungewichtet }}$ & 370 & 383 & 171 & 924 \\
\hline \multirow{9}{*}{$\begin{array}{l}\text { Sozial- } \\
\text { status }^{\mathrm{a}}\end{array}$} & Niedrig & $20,6 \%$ & $22,2 \%$ & $23,8 \%$ & $21,9 \%$ \\
\hline & $95 \%-\mathrm{Kl}$ & $15,0-27,7 \%$ & $15,9-30,0 \%$ & $15,6-34,5 \%$ & $18,1-26,4 \%$ \\
\hline & $\mathrm{n}_{\text {ungewichtet }}$ & 57 & 60 & 31 & 148 \\
\hline & Mittel & $53,3 \%$ & $58,5 \%$ & $61,9 \%$ & $57,3 \%$ \\
\hline & $95 \%-\mathrm{KI}$ & $45,5-60,9 \%$ & $51,3-65,3 \%$ & $51,6-71,2 \%$ & $52,8-61,6 \%$ \\
\hline & $\mathrm{n}_{\text {ungewichtet }}$ & 198 & 219 & 102 & 519 \\
\hline & Hoch & $26,1 \%$ & $19,3 \%$ & $14,3 \%$ & $20,8 \%$ \\
\hline & $95 \%-\mathrm{KI}$ & $20,2-33,1 \%$ & $15,4-24,0 \%$ & $9,2-21,6 \%$ & $17,5-24,5 \%$ \\
\hline & $\mathrm{n}_{\text {ungewichtet }}$ & 110 & 94 & 33 & 237 \\
\hline
\end{tabular}

tion ohne Prothese, Lähmungen, bei Personen im Rollstuhl oder nach einer aktuellen Operation an den unteren Extremitäten. Kann die Position einer Teststufe nicht $10 \mathrm{~s}$ lang gehalten werden, entfallen die nachfolgenden Teststufen. Für die Auswertungen werden je nach gehaltenen Tests zwischen 0 (Romberg-Stand nicht absolviert) und 5 Punkte (alle Positionen $10 \mathrm{~s}$ gehalten) nach dem FICSITProtokoll [36] vergeben. Eine verminderte Balancefähigkeit ist ein prognostisch bedeutender Faktor für zukünftige Stürze und Frakturen [23, 37], Einschränkungen der Mobilität [33] und Behinderung im Alltag [1].

\section{Greifkraft-Test}

Die isometrische Handgriffstärke wurde in DEGS1 mit dem Smedley Dynamometer (Scandidact, Denmark, $100 \mathrm{~kg}$ ) gemessen. Das Dynamometer wird an die Handgröße angepasst. Die Messung erfolgt, wenn möglich, im Stehen, der Oberarm liegt am Oberkörper an, der
Unterarm wird nach vorn angehoben, sodass er im rechten Winkel zum Oberarm gehalten wird. Es werden jeweils 2 Messungen abwechselnd rechts und links durchgeführt, dabei wird das Gerät mit maximaler Kraft für etwa $5 \mathrm{~s}$ zusammengedrückt. Die Messwerte werden auf 0,5 kg genau abgelesen. Die Messung wird bei starken Schmerzen, Operationen oder Verletzungen innerhalb der letzten 6 Monate an Fingern, Händen und Armen, ebenso bei Amputationen oder Lähmungen sowie bei Vorliegen von akuten Schwellungen, Entzündungen oder Verletzungen nicht durchgeführt. Falls nur eine Seite betroffen ist, wird die Messung nur an der nicht betroffenen Hand durchgeführt. Bewertet wird die maximal erreichte Greifkraft, unabhängig von Seite, Reihenfolge und Körperposition. In zahlreichen Studien zeigt sich die Handgreifkraft als einfach zu messender, objektiver Indikator für den Gesundheitszustand und als prognostischer Faktor für zukünftige gesund- heitliche Einschränkungen wie Behinderungen, Muskelschwäche oder Verlust der unabhängigen Lebensführung und erhöhte Sterblichkeit [2, 8, 38, 39]. Die Beziehung ist kontinuierlich. Eine Adjustierung der Messwerte für Geschlecht sowie für Größe, Gewicht oder Body-MassIndex ist notwendig [8]. Darüber hinaus besteht Konsens, dass Messwerte von weniger als $20 \mathrm{~kg}$ bei Frauen und weniger als $30 \mathrm{~kg}$ bei Männern ein Indikator für Sarkopenie sind [40].

\section{Zahlen-Symbol-Test}

Die kognitive Leistungsfähigkeit hat integrale Bedeutung für Alltagskompetenz, Autonomie, soziale Teilhabe und Lebensqualität im Alter. Einschränkungen in der kognitiven Leistungsfähigkeit stehen mit Alltagsbehinderung, Sturzrisiko und erhöhter Mortalität in Zusammenhang [28, 29, 41, 42].

Zur Beurteilung der kognitiven Leistungsfähigkeit wird in DEGS1 der Zahlen-Symbol-Test (ZST) aus dem Wechsler Intelligenz Test für Erwachsene (WIE) [27] eingesetzt. Der Zahlen-Symbol-Test ist ein in epidemiologischen und klinischen Studien vielfach eingesetzter Screening-Test zur unspezifischen Beurteilung der kognitiven Leistungsfähigkeit. Der Test erfordert eine Integration komplexer neuropsychologischer Prozesse. Dementsprechend werden mehrere Bereiche der kognitiven Funktion erfasst, insbesondere kognitive und psychomotorische Geschwindigkeit, Aufmerksamkeit, visuelle Suche und exekutive Funktionen. ${ }^{2}$

Der Zahlen-Symbol-Test ist ein geschwindigkeitsabhängiger Zuordnungstest, bei dem die Teilnehmenden einfache grafische Symbole nachzeichnen, die mit den Zahlen 1 bis 9 gepaart sind. Dazu ist auf dem Testbogen ein Zuordnungsschlüssel abgedruckt, der den Zahlen die grafischen Symbole zuordnet. Darunter sind in 7 Reihen mit jeweils 20 Feldern die Zahlen 1 bis 9 in zufälliger Abfolge vorgegeben. Unter diese Zahlen zeichnen die Teilnehmenden das jeweils zugehörige Symbol. Im Rahmen der Test-

\footnotetext{
2 Kognitive Prozesse, die für die Planung und Steuerung von Handlungen und für die Selbstregulation erforderlich sind.
} 
Tab. 2 Teilnahme und Nichtteilnahmegründe an den Funktionstests, differenziert nach Gründen in \% ( $\mathrm{n}=1853$ )

\begin{tabular}{|c|c|c|c|c|c|c|c|c|c|}
\hline & \multirow{2}{*}{$\begin{array}{l}\text { Timed-up } \\
\text { and Go- } \\
\text { Test }\end{array}$} & \multirow{2}{*}{$\begin{array}{l}\text { Chair- } \\
\text { Rise-Test }\end{array}$} & \multicolumn{5}{|c|}{ Balancetests } & \multirow{2}{*}{$\begin{array}{l}\text { Greif- } \\
\text { kraft-Test }\end{array}$} & \multirow{2}{*}{$\begin{array}{l}\text { Zahlen- } \\
\text { Symbol- } \\
\text { Test }\end{array}$} \\
\hline & & & $\begin{array}{l}\text { Rom- } \\
\text { berg-Test }\end{array}$ & $\begin{array}{l}\text { Semitan- } \\
\text { dem-Test }\end{array}$ & $\begin{array}{l}\text { Tandem- } \\
\text { Test }\end{array}$ & $\begin{array}{l}\text { Einbein- } \\
\text { stand rechts }\end{array}$ & $\begin{array}{l}\text { Einbein- } \\
\text { stand links }\end{array}$ & & \\
\hline $\begin{array}{l}\text { Am jeweiligen Test teilge- } \\
\text { nommen }\end{array}$ & $97,2 \%$ & $90,7 \%$ & $96,0 \%$ & $95,6 \%$ & $89,4 \%$ & $73,2 \%$ & $73,1 \%$ & $95,1 \%$ & $96,7 \%$ \\
\hline 95\%-KI & $\begin{array}{l}95,3- \\
98,4 \% \\
\end{array}$ & $\begin{array}{l}88,5- \\
92,6 \%\end{array}$ & $\begin{array}{l}94,1- \\
97,3 \%\end{array}$ & $\begin{array}{l}93,7- \\
97,0 \%\end{array}$ & $\begin{array}{l}86,9- \\
91,5 \%\end{array}$ & $\begin{array}{l}69,8- \\
76,4 \% \\
\end{array}$ & $\begin{array}{l}69,6- \\
76,2 \% \\
\end{array}$ & $\begin{array}{l}92,9- \\
96,6 \%\end{array}$ & $\begin{array}{l}94,7- \\
98,0 \%\end{array}$ \\
\hline $\mathrm{n}_{\text {ungewichtet }}$ & 1795 & 1675 & 1774 & 1769 & 1667 & 1410 & 1406 & 1774 & 1802 \\
\hline $\begin{array}{l}\text { Aus gesundheitlichen Grün- } \\
\text { den nicht teilgenommen }{ }^{\mathrm{a}}\end{array}$ & $1,1 \%$ & $1,7 \%$ & $2,3 \%$ & $2,3 \%$ & $4,3 \%$ & $5,7 \%$ & $5,4 \%$ & $2,7 \%$ & $1,4 \%$ \\
\hline 95\%-KI & $0,7-1,8 \%$ & $1,0-2,7 \%$ & $1,6-3,3 \%$ & $1,6-3,3 \%$ & $3,1-5,8 \%$ & $4,4-7,4 \%$ & $4,2-7,0 \%$ & $1,7-4,3 \%$ & $0,9-2,4 \%$ \\
\hline $\mathrm{n}_{\text {ungewichtet }}$ & 22 & 33 & 43 & 46 & 77 & 99 & 97 & 40 & 21 \\
\hline $\begin{array}{l}\text { Aus persönlichen Gründen } \\
\text { nicht teilgenommen }{ }^{\text {b }}\end{array}$ & $0,6 \%$ & $1,4 \%$ & $0,7 \%$ & $0,8 \%$ & $4,0 \%$ & $6,3 \%$ & $6,3 \%$ & $0,6 \%$ & $0,7 \%$ \\
\hline 95\%-KI & $0,2-1,8 \%$ & $0,8-2,5 \%$ & $0,3-1,8 \%$ & $0,3-1,8 \%$ & $2,9-5,5 \%$ & $4,9-8,1 \%$ & $5,0-8,1 \%$ & $0,2-1,8 \%$ & $0,3-1,9 \%$ \\
\hline $\mathrm{n}_{\text {ungewichtet }}$ & 5 & 20 & 7 & 8 & 57 & 99 & 101 & 5 & 6 \\
\hline $\begin{array}{l}\text { Aus technischen/organisato- } \\
\text { rischen Gründen nicht teilge- } \\
\text { nommen }^{c}\end{array}$ & $0,8 \%$ & $0,8 \%$ & $0,7 \%$ & $0,7 \%$ & $0,8 \%$ & $0,8 \%$ & $0,8 \%$ & $0,8 \%$ & $0,6 \%$ \\
\hline 95\%-KI & $0,2-3,2 \%$ & $0,2-3,2 \%$ & $0,2-3,2 \%$ & $0,2-3,2 \%$ & $0,2-3,1 \%$ & $0,2-3,1 \%$ & $0,2-3,1 \%$ & $0,2-3,0 \%$ & $0,1-3,5 \%$ \\
\hline $\mathrm{n}_{\text {ungewichtet }}$ & 24 & 24 & 22 & 22 & 23 & 23 & 23 & 23 & 17 \\
\hline Voraussetzungen nicht erfüllt ${ }^{d}$ & & $4,4 \%$ & & $0,3 \%$ & $1,2 \%$ & $13,5 \%$ & $13,5 \%$ & & \\
\hline 95\%-KI & & $3,3-5,8 \%$ & & $0,0-1,8 \%$ & $0,6-2,1 \%$ & $11,2-16,3 \%$ & $11,2-16,3 \%$ & & \\
\hline $\mathrm{n}_{\text {ungewichtet }}$ & & 84 & & 1 & 21 & 213 & 213 & & \\
\hline $\begin{array}{l}\text { Ohne Angabe von Gründen } \\
\text { nicht durchgeführt }\end{array}$ & $0,3 \%$ & $1,0 \%$ & $0,3 \%$ & $0,3 \%$ & $0,4 \%$ & $0,4 \%$ & $0,8 \%$ & $0,9 \%$ & $0,6 \%$ \\
\hline 95\%-KI & $0,1-0,8 \%$ & $0,6-1,8 \%$ & $0,1-0,8 \%$ & $0,1-0,8 \%$ & $0,2-0,9 \%$ & $0,2-0,9 \%$ & $0,4-1,9 \%$ & $0,4-2,1 \%$ & $0,2-1,5 \%$ \\
\hline $\mathrm{n}_{\text {ungewichtet }}$ & 7 & 17 & 7 & 7 & 8 & 9 & 13 & 11 & 7 \\
\hline
\end{tabular}

anweisung werden die ersten 7 Felder als Beispielaufgaben bearbeitet. Der Testwert ergibt sich aus der Anzahl der Symbole, die innerhalb von $120 \mathrm{~s}$ in den übrigen Feldern nacheinander richtig eingetragen werden. Hierbei können somit maximal 133 Punkte erreicht werden.

Ausschlussgründe für den ZahlenSymbol-Test sind höhergradige Sehbehinderungen ohne Korrekturmöglichkeit und Beeinträchtigungen der Schreibfähigkeit bzw. der Handmotorik (z. B. durch Lähmung, schwere Gelenkerkrankungen, aktuelle Operation, Fraktur oder andere lokale Erkrankung).

Eine Bewertung des individuellen Testergebnisses kann anhand der publizierten altersspezifischen Normwerte aus der deutschsprachigen Normierungsstichprobe des WIE vorgenommen werden [27].

\section{Sozialstatus}

Der Sozialstatus wurde anhand eines Indexes bestimmt, in den Angaben zu schulischer und beruflicher Ausbildung, beruflicher Stellung sowie Haushaltsnettoeinkommen (bedarfsgewichtet) eingehen und der eine Einteilung in niedrige, mittlere und hohe Statusgruppe ermöglicht [43].

\section{Statistische Auswertung}

Die Ergebnisse zu den einzelnen Funktionstests wurden mit 95\%-Konfidenzintervall (95\%-KI) nach Geschlecht und Altersgruppe berechnet. Personen mit fehlenden Angaben wurden von den Analysen ausgeschlossen. Unterschiede zwischen den Gruppen wurden in linearen Regressionsanalysen untersucht.

Die Querschnittanalysen wurden mit einem Gewichtungsfaktor durchgeführt, der Abweichungen der Stich- probe von der Bevölkerungsstruktur (Stand 31.12.2010) hinsichtlich Alter, Geschlecht, Region und Staatsangehörigkeit sowie Gemeindetyp und Bildung korrigiert [14]. Ferner wurde bei den ehemaligen Teilnehmerinnen und Teilnehmern des BGS98 die unterschiedliche Wiederteilnahmebereitschaft ausgeglichen. Für den Untersuchungsteil wurde ein gesonderter Gewichtungsfaktor erstellt. Bei der Berechnung der Gewichtung für die ehemaligen Teilnehmenden des BGS98 wurde die Wiederteilnahmewahrscheinlichkeit, basierend auf einem logistischen Modell, berücksichtigt. Eine Nonresponder-Analyse und der Vergleich einzelner erhobener Indikatoren mit Daten der amtlichen Statistik weisen auf eine hohe Repräsentativität der Stichprobe für die Wohnbevölkerung in Deutschland hin [14]. Um sowohl die Gewichtung als auch die Korrelation der Teilnehmenden innerhalb einer Gemeinde zu berücksichtigen, wurden die Konfidenzin- 
Tab. 3 Ergebnisse der Funktionstests (Mittelwerte und 95\%-Konfidenzintervalle)

\begin{tabular}{|c|c|c|c|c|c|}
\hline \multirow[t]{2}{*}{ Test } & & \multicolumn{3}{|l|}{ Altersgruppe } & \multirow[t]{2}{*}{ Gesamt } \\
\hline & & 65 bis 69 Jahre & 70 bis 74 Jahre & 75 bis 79 Jahre & \\
\hline \multirow{6}{*}{$\begin{array}{l}\text { Timed-up and } \\
\text { Go-Test (TUG; } \\
\text { benötigte Zeit } \\
\text { in Sekunden) } \\
n=1795\end{array}$} & Frauen & 10,3 & 11,0 & 12,0 & 11,0 \\
\hline & 95\%-KI & $9,9-10,7$ & $10,5-11,4$ & $11,3-12,7$ & $10,7-11,3$ \\
\hline & Männer & 9,7 & 10,5 & 11,4 & 10,4 \\
\hline & $95 \%-\mathrm{KI}$ & $9,4-10,1$ & $9,9-11,0$ & $10,7-12,1$ & $10,0-10,7$ \\
\hline & Gesamt & 10,0 & 10,7 & 11,8 & 10,7 \\
\hline & 95\%-KI & $9,7-10,3$ & $10,4-11,1$ & $11,2-12,3$ & $10,4-11,0$ \\
\hline \multirow{6}{*}{$\begin{array}{l}\text { Chair-Rise-Test } \\
\text { (benötigte Zeit } \\
\text { in Sekunden) } \\
n=1675\end{array}$} & Frauen & 11,8 & 12,1 & 12,6 & 12,1 \\
\hline & $95 \%-\mathrm{KI}$ & $11,0-12,7$ & $11,6-12,7$ & $12,0-13,2$ & $11,7-12,6$ \\
\hline & Männer & 10,7 & 11,0 & 13,1 & 11,3 \\
\hline & 95\%-KI & $10,2-11,2$ & $10,6-11,4$ & $11,9-14,4$ & $10,9-11,7$ \\
\hline & Gesamt & 11,3 & 11,6 & 12,8 & 11,8 \\
\hline & 95\%-KI & $10,8-11,8$ & $11,2-12,0$ & $12,1-13,5$ & $11,4-12,1$ \\
\hline \multirow{6}{*}{$\begin{array}{l}\text { Balance-Test } \\
\text { (Punktwert } \\
\text { nach FICSIT4- } \\
\text { Protokoll) } \\
n=1774\end{array}$} & Frauen & 4,1 & 3,9 & 3,2 & 3,8 \\
\hline & 95\%-KI & $4,0-4,3$ & $3,7-4,0$ & $3,0-3,4$ & $3,7-3,9$ \\
\hline & Männer & 4,3 & 4,0 & 3,6 & 4,0 \\
\hline & 95\%-KI & $4,1-4,5$ & $3,8-4,2$ & $3,4-3,8$ & $3,9-4,1$ \\
\hline & Gesamt & 4,2 & 3,9 & 3,4 & 3,9 \\
\hline & 95\%-KI & $4,1-4,3$ & $3,8-4,0$ & $3,2-3,5$ & $3,8-4,0$ \\
\hline \multirow{6}{*}{$\begin{array}{l}\text { Greifkraftmes- } \\
\text { sung (mittlere } \\
\text { Greifkraft in kg) } \\
n=1774\end{array}$} & Frauen & 26,0 & 25,2 & 23,2 & 25,0 \\
\hline & $95 \%-\mathrm{KI}$ & $25,3-26,7$ & $24,6-25,7$ & $22,5-24,0$ & $24,6-25,4$ \\
\hline & Männer & 42,5 & 40,8 & 36,5 & 40,5 \\
\hline & $95 \%-\mathrm{KI}$ & $41,7-43,4$ & $39,6-42,0$ & $35,4-37,6$ & $39,8-41,3$ \\
\hline & Gesamt & 34,1 & 32,5 & 29,0 & 32,3 \\
\hline & 95\%-KI & $33,2-35,0$ & $31,6-33,4$ & $28,1-30,0$ & $31,7-32,9$ \\
\hline \multirow{6}{*}{$\begin{array}{l}\text { Zahlen-Symbol- } \\
\text { Test (Anzahl } \\
\text { korrekter Zei- } \\
\text { chen) } \\
n=1802\end{array}$} & Frauen & 51,4 & 43,1 & 38,4 & 44,8 \\
\hline & 95\%-KI & $48,6-54,2$ & $41,3-44,9$ & $36,6-40,3$ & $43,4-46,3$ \\
\hline & Männer & 46,3 & 41,7 & 38,0 & 42,6 \\
\hline & 95\%-KI & $44,4-48,2$ & $39,9-43,3$ & $35,0-40,9$ & $41,3-43,9$ \\
\hline & Gesamt & 48,9 & 42,4 & 38,2 & 43,8 \\
\hline & 95\%-KI & $47,2-50,7$ & $41,3-43,6$ & $36,7-39,8$ & $42,8-44,8$ \\
\hline
\end{tabular}

tervalle mit den Verfahren für komplexe Stichproben von SPSS-20 bestimmt. Unterschiede werden als statistisch signifikant angesehen, wenn sich die jeweiligen 95\%-Konfidenzintervalle nicht überschneiden oder wenn sich in Regressionsanalysen ein $\mathrm{p}$-Wert von kleiner als 0,05 ergibt.

\section{Ergebnisse}

- Tab. 1 gibt die Charakteristika der Studienpopulation für die Funktionstests wieder. Von den 1853 Personen zwischen 65 und 79 Jahren, die das Untersuchungszentrum besuchten, waren 53,9\% Frauen, 46,1\% Männer. Weniger als ein Viertel war zwischen 75 und 79 Jahre alt. Die Mehrzahl der Teilnehmenden war dem mittleren Sozialstatus zuzu- ordnen (Frauen 62,2\%, Männer 57,3\%), 28,1\% der Frauen und 21,9\% der Männer dem niedrigen und 9,1\% der Frauen und 20,8\% der Männer dem hohen; Frauen weisen signifikant seltener einen hohen Sozialstatus auf als Männer.

Die Teilnahmerate an den verschiedenen Funktionstests variiert, bedingt durch unterschiedliche Ein- und Ausschlussgründe. - Tab. 2 zeigt die Teilnahmeraten und schlüsselt die Nichtteilnahmegründe auf. Häufigste Nichtteilnahmegründe sind bei allen Tests gesundheitliche Gründe, hier in erster Linie neurologische oder orthopädische Einschränkungen oder testrelevante Erkrankungen. Da sich Frauen und Männer hinsichtlich des Teilnahmeverhaltens bei keinem der Funktionstests signifikant unterscheiden, wird hier auf eine geschlechtergetrennte Darstellung der Ergebnisse verzichtet.

- Tab. 3 fasst die Ergebnisse für die durchgeführten Funktionstests zusammen.

\section{Körperliche Funktionsfähigkeit}

\section{Timed-up and Go-Test (TUG)}

97,2\% der Personen zwischen 65 und 79 Jahren nahmen am TUG teil. Die genannten Gründe für eine Nichtteilnahme am TUG sind vorwiegend gesundheitliche Gründe (1,1\%) (• Tab. 2).

Im Durchschnitt benötigen die Teilnehmenden 10,7 s für die Absolvierung des TUG-Tests, der Median beträgt $10 \mathrm{~s}$. Die Spannweite der benötigten Zeit reicht von 4,6 bis 46,3 s. Die Mittelwerte liegen bei Frauen bei 11,0 s und bei Männern bei $10,4 \mathrm{~s}$, diese Unterschiede sind statistisch nicht signifikant $(\mathrm{p}=0,129)$, wenn neben Geschlecht das Alter und der Sozialstatus berücksichtigt werden. Mit zunehmendem Alter benötigten alle Teilnehmenden signifikant länger für die Durchführung des TUG.

Von den insgesamt 1853 Personen, die ins Untersuchungszentrum kamen, konnten 24 aus organisatorischen Gründen nicht am TUG teilnehmen. Von der verbleibenden 1829 Personen, die potenziell am TUG teilnehmen konnten, gibt es bei 2,0\% Hinweise auf eine alltagsrelevante Gehstörung, d. h., die benötigte Zeit für die Durchführung des TUG beträgt $20 \mathrm{~s}$ und mehr. Mobilitätseinschränkungen liegen größtenteils bei den $1,1 \%$ vor, die aus gesundheitlichen Gründen nicht teilnehmen konnten.

\section{Chair-Rise-Test}

90,7\% der Personen zwischen 65 und 79 Jahren nahmen am Chair-Rise-Test teil. 4,4\% der Personen konnten den Test nicht durchführen, da ein einmaliges Aufstehen nicht möglich war. Weitere $1,7 \%$ nahmen aus gesundheitlichen Gründen nicht teil, hier vor allem wegen gesundheitlicher Einschränkungen (• Tab. 2).

Im Mittel wird das fünfmalige Aufstehen in 11,8 s bewältigt, der Median liegt bei $11,0 \mathrm{~s}$. Die kürzeste benötigte Zeit beträgt $4,0 \mathrm{~s}$, die längste $41,2 \mathrm{~s}$. Frauen benötigen für den Chair-Rise-Test nach 


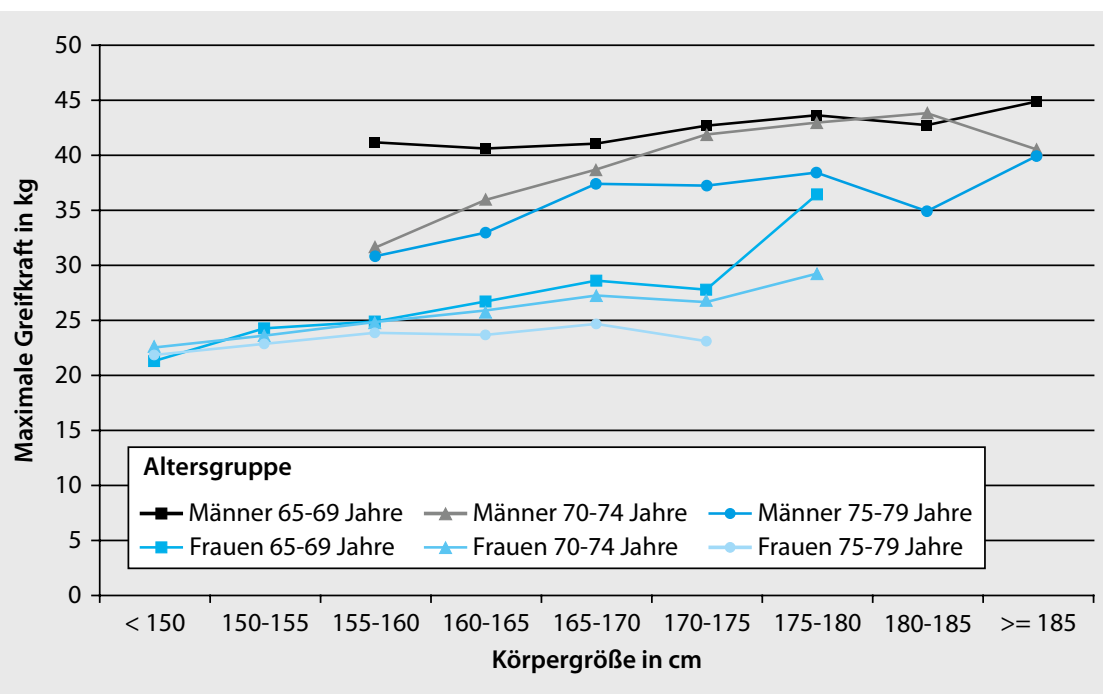

Abb. $2 \Delta$ Mittlere maximale Greifkraft nach Körpergröße

Adjustierung für Alter und Sozialstatus mit 12,1 s signifikant mehr Zeit als

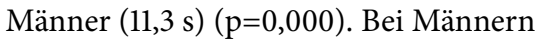
zeigt sich darüber hinaus ein signifikanter Einfluss des Alters ( $\mathrm{p}=0,001)$.

\section{Balance-Tests}

Bei den Balance-Tests ist die im Testablauf angelegte Steigerung der Schwierigkeit (Teilnehmende absolvieren die nächstschwierigere Stufe nur, falls sie den vorhergehenden leichteren Test schaffen) sichtbar. Der Anteil an Personen, die einzelne Tests nicht durchführen konnten, steigt vom Romberg-Stand bis zum Einbeinstand (rechts) von 3,3 auf $25,5 \%$ an (- Tab. 2). Gesundheitliche Gründe, die zu einer Nichtteilnahme an einem oder mehreren Balancetests führten, waren akut erhöhter Blutdruck, eingeschränkte Gehfähigkeit, künstliche Gelenke und neurologische oder orthopädische Probleme an den Beinen.

Von den maximal möglichen 5 Punkten auf der FICSIT4-Balance-Skala erreichen die Teilnehmenden durchschnittlich 3,9 Punkte, der Median liegt bei 4 Punkten. Die Spannweite reicht von 0 bis 5 Punkten. Es gibt keinen signifikanten Unterschied in der Balancefähigkeit zwischen den Geschlechtern ( $\mathrm{p}=0,08)$. Sie nimmt bei beiden Geschlechtern nach Adjustierung für Sozialstatus mit zunehmendem Alter signifikant ab; bei Frauen sinkt sie von 4,1 Punkten bei den 65- bis 69-Jährigen auf 3,2 Punkte bei den 75- bis 79-Jährigen, bei den Männern sinkt der Wert von 4,3 bei den 65bis 69 -Jährigen auf 3,6 bei den 75 - bis 79-Jährigen.

\section{Greifkraft-Test}

Der Greifkraft-Test wurde von 95,1\% der Teilnehmenden durchgeführt. Starke Schmerzen, Verletzungen und Operationen an den Händen und akute Entzündungen und Schwellungen waren die häufigsten Gründe für eine Nichtteilnahme.

Der Mittelwert der maximalen Greifkraft aus den 4 Greifkraftmessungen liegt für die 65- bis 79-Jährigen bei $32,3 \mathrm{~kg}$, der Median bei 31,0 kg. Männer und Frauen unterscheiden sich adjustiert nach Sozialstatus signifikant in der Greifkraft; Frauen liegen im Mittelwert bei 25,0 kg, Männer bei $40,5 \mathrm{~kg}(\mathrm{p}<0,001)$. Mit zunehmendem Alter nimmt bei beiden Geschlechtern die Greifkraft signifikant ab.

Die Verteilung der maximalen Greifkraft (arithmetisches Mittel) unter Berücksichtigung der Körpergröße zeigt

- Abb. 2. Es wird deutlich, dass, abgesehen von der ältesten Altersgruppe, die Greifkraft mit zunehmender Körpergröße zunimmt (• Abb. 1).

Eine stark verringerte Muskelkraft $(<20 \mathrm{~kg}$ bei Frauen und $<30 \mathrm{~kg}$ bei Männern) zeigt sich bei 9,5\% der Frauen und $5,1 \%$ der Männer im Alter von 65 bis 79 Jahren und kann auf eine Sarkopenie hinweisen.

\section{Kognitive Leistungsfähigkeit}

Der Zahlen-Symbol-Test wurde von 96,7\% der Teilnehmenden durchgeführt. Gesundheitliche Einschränkungen waren der häufigste Ausschlussgrund, wobei durchweg Beeinträchtigungen der Handmotorik oder Sehbehinderungen vorlagen.

Insgesamt werden Testwerte zwischen 0 und 96 Punkten erreicht, die Testwerte sind normalverteilt. Der durchschnittliche Testwert liegt bei 43,8 Punkten (Standardabweichung: 14,0). Frauen erreichen insgesamt höhere Testwerte (Mittelwert: 44,8) als Männer (Mittelwert: 42,6$)(\mathrm{p}=0,02)$. Mit zunehmendem Alter sinkt der durchschnittliche Testwert kontinuierlich ab. Dieser Alterseffekt besteht gleichermaßen bei Frauen und Männern. Dabei erreichen Frauen in der Altersgruppe der 65- bis 69-Jährigen, nicht aber in den anderen Altersgruppen, höhere durchschnittliche Testwerte. Ein höherer sozioökonomischer Status geht ebenfalls mit höheren Testwerten einher, auch wenn für den Einfluss von Alter und Geschlecht statistisch kontrolliert wird.

\section{Diskussion}

Die Querschnittergebnisse aus DEGS1 zeigen die erwartete altersassoziierte Abnahme der Funktionsfähigkeit. Obwohl Alter nicht die alleinige Determinante für den Verlust der Funktionsfähigkeit darstellt, spielen altersphysiologische Prozesse eine wichtige Rolle. Die Abnahme der Leistungsfähigkeit mit zunehmendem Alter ist multifaktoriell bedingt. Neben genetischen Faktoren sind insbesondere über die Lebenszeit einwirkende individuelle Verhaltensweisen (Ernährung, Bewegung, Rauchen) und Umwelteinflüsse entscheidende Faktoren für altersassoziierte Veränderungen auf zellulärer und Organebene. Dies erklärt, warum altersassoziierte Einschränkungen der körperlichen und kognitiven Funktionseinschränkungen große interindividuelle Variabilität aufweisen $[44,45,46]$. Hieraus ergibt sich die besondere Bedeutung von Prävention und Gesundheitsförderung im höheren Lebensalter. 
Die Ergebnisse aus DEGS1 sind vergleichbar mit Daten aus verschiedenen internationalen Publikationen (s. unten). Bei der Einordnung der Ergebnisse ist allerdings zu berücksichtigen, dass sich die Altersgruppen, Altersspannen oder Gesundheitszustand (gesund, beeinträchtigt) zum Teil erheblich unterscheiden.

Für den TUG berichtet Bohannon [47] in einer Metaanalyse 9,4 s für die 60 - bis 99-Jährigen und eine Zunahme der benötigten Zeit mit ansteigendem Alter. Die Metaanalyse zeigt allerdings auch eine große Spannweite der Ergebnisse. Rockwood et al. [48] ermitteln in einer repräsentativen kanadischen Stichprobe von Personen im Alter von 69 bis 104 Jahren eine mittlere benötigte Zeit von 14,0 s (Median).

Die in DEGS1 benötigte durchschnittliche Zeit von 10,7 s liegt zwischen den beiden Werten und entspricht in Anbetracht der Altersstruktur den genannten Ergebnissen.

Auch Ergebnisse zum Chair-Rise-Test differieren aufgrund der unterschiedlichen Altersgruppierungen in den Studien. Guralnik et al. [22] berichten 14,5 s für Personen ab 71 Jahre ohne Altersobergrenze, Whitney et al. [49] 13,4 s bei einem durchschnittlichen Alter von 73 Jahren. In einer weiteren Metaanalyse von Bohannon [50] werden 11,4 s für 60 - bis 69 -Jährige und 12,6 s für 70 bis 79-Jährige berichtet. Die in DEGS1 durchschnittlich benötigten $11,8 \mathrm{~s}$ liegen in diesem Bereich. Altersgruppierte Werte finden sich im Health Survey for England [51]: die mittlere benötigte Zeit beträgt bei den 60 - bis 69 -jährigen Frauen 13 s, bei Männern $12 \mathrm{~s}$ und steigt an auf 15 bzw. 14 s bei den 75 - bis 79-Jährigen. Entsprechende Alters- und Geschlechtsunterschiede finden sich in DEGS1.

Die in DEGS1 beschriebene Abnahme der Balancefähigkeit mit zunehmendem Alter entspricht den Ergebnissen anderer Studien [22, 36, 51]. Geplant sind Auswertungen zum bekannten Zusammenhang zwischen Balancefähigkeit und Stürzen [23] und Mobilitätseinschränkungen [33].

In Deutschland wurde die Messung der Handgreifkraft erstmalig 2004 im
Rahmen des Survey of Health, Ageing and Retirement in Europe (SHARE) und der Haupterhebung 2006 des Soziooekonomischen Panels (SOEP) eingesetzt; die Ergebnisse belegen einen deutlich positiven Zusammenhang zwischen Handgreifkraft und Gesundheit [8]. Die in DEGS1 ermittelten Werte zeigen eine gute Übereinstimmung mit SOEP und SHARE-Daten.

Längsschnittstudien haben gezeigt, dass Muskelschwäche im mittleren Lebensalter, gemessen über die isometrische Greifkraft, ein sehr guter Prädiktor für zukünftige Behinderungen, etwa bei den Aktivitäten des täglichen Lebens (ADL) [52] oder für Mortalitätsrisiken im höheren Alter [53] ist.

Sowohl die Greifkraft als auch die Ergebnisse des Chair-Rise-Tests gelten als Indikatorvariable für abnehmende Muskelkraft, ein Anzeichen für Sarkopenie. Mit DEGS1 ergibt sich somit die Möglichkeit, eine Schätzung der von Sarkopenie Betroffenen in der Allgemeinbevölkerung im Zusammenhang mit ihrer körperlichen und sportlichen Aktivität vorzunehmen.

Zum Zahlen-Symbol-Test liegen altersspezifische Normwerte für eine gesunde deutschsprachige Normstichprobe von insgesamt 1897 Personen aus Deutschland, Österreich und der deutschsprachigen Schweiz vor, darunter 421 Personen im Alter zwischen 65 und 79 Jahren [27]. In dieser Normstichprobe lagen die Vertrauensbereiche der mittleren Testwerte bei $48-53$ bei den 65 - bis 69-Jährigen, bei $44-49$ bei den 70- bis 74-Jährigen und bei 39-44bei den 75- bis 79-Jährigen. Erwartungsgemäß liegen die in DEGS1 gemessenen mittleren Testwerte in allen Altersgruppen sehr nahe an der jeweils unteren Grenze dieser Vertrauensbereiche.

Dieses Ergebnis ist plausibel, da sich die hier vorgestellten Ergebnisse auf die gesamte DEGS1-Querschnittstichprobe der 65- bis 79-Jährigen beziehen, die keine Normstichprobe darstellt. Somit flossen im Unterschied zu der oben genannten Normstichprobe in die hier vorgestellte Auswertung auch Testwerte von Personen mit krankheitsbedingt reduzierter kognitiver Leistungsfähigkeit (z. B. nach einer Hirnerkrankung) ein.
Über den Alterseffekt hinaus zeigt sich in den vorgestellten Ergebnissen bereits ein Zusammenhang der mittleren Testwerte mit dem Geschlecht und dem sozioökonomischen Status, wie er für ähnliche Zuordnungstests beschrieben wurde, z. B. für den Letter-DigitSubstitution-Test [54].

\section{Fazit und Ausblick}

Die vorliegende Auswertung liefert erste Anhaltspunkte zur Funktionsfähigkeit in der Bevölkerung zwischen 65 und 79 Jahren. Vertiefende Auswertungen werden zum einen dazu dienen, die Verteilung von Funktionsmesswerten nach anthropometrischen und soziodemografischen Einflussfaktoren zu analysieren. Ein weiterer Schwerpunkt wird in der Erstellung von Normverteilungen liegen. Bisher fehlen für Deutschland z. B. alters-, geschlechtsund schichtspezifische Normverteilungen zur Handgreifkraft unter Berücksichtigung von Körpergröße und -gewicht sowie aktuelle alters-, geschlechts- und bildungsgruppenspezifische Normwerte zum Zahlen-SymbolTest für 65- bis 79-Jährige in Deutschland. Neben Auswertungen von einzelnen körperlichen Funktionstests werden häufig darauf basierende Gesamtwertungen (Scores) zur körperlichen Funktionsfähigkeit gebildet [35], die in Zusammenhang mit Mortalität, Stürzen, Behinderung oder Hospitalisierung betrachtet werden. Angelehnt an die SPPB [22] ist geplant, anhand der DEGS1-Ergebnisse einen Index zu bilden, der die Gesamtfähigkeit auf Basis mehrerer Funktionen erfasst und Zusammenhangsanalysen ermöglicht. Darüber hinaus wird der Zusammenhang zwischen Funktionseinschränkungen und Erkrankungen, Frakturen, Krankenhausaufenthalten, Lebensqualität und Behinderung im Alltag untersucht werden. Die Längsschnittkomponente von DEGS eröffnet zudem die Möglichkeit der Analyse von Entwicklungen im Lebensverlauf. Diese Fragestellungen sind auch im Hinblick auf den Austausch von Daten im internationalen Vergleich relevant $[2,3]$. 
Die im Rahmen des Gesundheitsmonitorings regelmäßig hierzu erhobenen Daten werden ein Monitoring zur Entwicklung von Krankheitslast und Versorgungsbedarf in einer älter werdenden Gesellschaft ermöglichen [9, 10, 11].

\section{Korrespondenzadresse}

\section{Dr. J. Fuchs}

Abteilung für Epidemiologie und Gesundheitsmonitoring, Robert Koch-Institut General-Pape-Str. 62-66, 12101 Berlin Fuchs」@rki.de

Finanzierung der Studie. Die Studie wurde finanziert mit Mitteln des Robert Koch-Instituts und des Bundesministeriums für Gesundheit.

Interessenkonflikt. Die korrespondierende Autorin gibt für sich und ihre Koautoren an, dass kein Interessenkonflikt besteht.

\section{Literatur}

1. Vermeulen J, Neyens JC, Rossum E van et al (2011) Predicting ADL disability in communitydwelling elderly people using physical frailty indicators: a systematic review. BMC Geriatr 11:33

2. Cooper R, Kuh D, Cooper C et al (2011) Objective measures of physical capability and subsequent health: a systematic review. Age Ageing 40:14-

3. Cooper R, Kuh D, Hardy R et al (2010) Objectively measured physical capability levels and mortality: systematic review and meta-analysis. BMJ 341:c4467

4. Hergenroeder AL, Wert DM, Hile ES et al (2011) Association of body mass index with self-report and performance-based measures of balance and mobility. Phys Ther 91:1223-1234

5. Roubenoff R (2000) Sarcopenia and its implications for the elderly. Eur J Clin Nutr 54(Suppl 3): 5 40-47

6. Beenakker KG, Ling CH, Meskers CG et al (2010) Patterns of muscle strength loss with age in the general population and patients with a chronic inflammatory state. Ageing Res Rev 9:431-436

7. VanSwearingen JM, Brach JS (2001) Making geriatric assessment work: selecting useful measures. Phys Ther 81:1233-1252

8. Hank K, Jurges H, Schupp J, Wagner GG (2009) Isometrische Greifkraft und sozialgerontologische Forschung. Ergebnisse und Analysepotentiale des SHARE und SOEP. Z Gerontol Geriatr 42:117-126

9. Saß AC, Wurm S, Scheidt-Nave C (2010) Alter und Gesundheit. Bundesgesundheitsbl Gesundheitsforsch Gesundheitsschutz 53:404-416

10. Parker MG, Thorslund M (2007) Health trends in the elderly population: getting better and getting worse. Gerontologist 47:150-158

11. Fries JF (2003) Measuring and monitoring success in compressing morbidity. Ann Intern Med 139:455-459
12. World Health Organisation (WHO) (2005) Internationale Klassifikation der Funktionsfähigkeit, Behinderung und Gesundheit.Genf

13. Gößwald A, Lange M, Kamtsiuris P, Kurth BM (2012) DEGS: Studie zur Gesundheit Erwachsener in Deutschland. Bundesweite Quer- und Längsschnittstudie im Rahmen des Gesundheitsmonitorings des Robert Koch-Instituts. Bundesgesundheitsbl Gesundheitsforsch Gesundheitsschutz 55:775-780

14. Kamtsiuris $P$, Lange $M$, Hoffmann R et al (2013) Die erste Welle der Studie zur Gesundheit Erwachsener in Deutschland (DEGS1). Stichprobendesign, Response, Gewichtung und Repräsentativität. Bundesgesundheitsbl Gesundheitsforsch Gesundheitsschutz 56:620-630

15. Kurth BM (2012) Das RKI-Gesundheitsmonitoring - was es enthält und wie es genutzt werden kann. Public Health Forum 20(76):4e1-4e3

16. Kurth BM, Lange $C$, Kamtsiuris $P$, Hölling $H$ (2009) Gesundheitsmonitoring am Robert Koch-Institut, Sachstand und Perspektiven. Bundesgesundheitsbl Gesundheitsforsch Gesundheitsschutz 52:557-570

17. Scheidt-Nave C, Kamtsiuris P, Gößwald A et al (2012) German Health Interview and Examination Survey for Adults (DEGS) - design, objectives and implementation of the first data collection wave. BMC Public Health 12:730

18. Gößwald A, Lange M, Dölle R, Hölling H (2013) Die erste Welle der Studie zur Gesundheit Erwachsener in Deutschland (DEGS1). Gewinnung von Studienteilnehmenden, Durchführung der Feldarbeit und Qualitätsmanagement. Bundesgesundheitsbl Gesundheitsforsch Gesundheitsschutz 56:611-619

19. Robert Koch-Institut (Hrsg) (2009) DEGS: Studie zur Gesundheit Erwachsener in Deutschland Projektbeschreibung. Beiträge zur Gesundheitsberichterstattung des Bundes. RKI, Berlin

20. Podsiadlo D, Richardson S (1991) The timed „Up \& Go": a test of basic functional mobility for frail elderly persons. J Am Geriatr Soc 39:142-148

21. Shumway-Cook A, Brauer S, Woollacott M (2000) Predicting the probability for falls in communitydwelling older adults using the Timed Up \& Go Test. Phys Ther 80:896-903

22. Guralnik JM, Simonsick EM, Ferrucci L et al (1994) A short physical performance battery assessing lower extremity function: association with selfreported disability and prediction of mortality and nursing home admission. J Gerontol 49:M85M94

23. Stevens KN, Lang IA, Guralnik JM, Melzer D (2008) Epidemiology of balance and dizziness in a national population: findings from the English longitudinal study of ageing. Age Ageing 37:300-305

24. Rantanen T, Volpato S, Ferrucci L et al (2003) Handgrip strength and cause-specific and total mortality in older disabled women: exploring the mechanism. J Am Geriatr Soc 51:636-641

25. Mohd Hairi F, Mackenbach JP, Andersen-Ranberg K, Avendano M (2010) Does socio-economic status predict grip strength in older Europeans? Results from the SHARE study in non-institutionalised men and women aged $50+$. J Epidemiol Community Health 64:829-837

26. Welmerink DB, Longstreth WT, Lyles MF, Fitzpatrick AL (2010) Cognition and the risk of hospitalization for serious falls in the elderly: results from the cardiovascular health study. J Gerontol A Biol Sci Med Sci 65A:1242-1249
27. Aster M, Neubauer AC, Horn R von (2006) Wechsler Intelligenztest fuer Erwachsene (WIE). Harcourt, Frankfurt a. M.

28. Sachs GA, Carter R, Holtz LR et al (2011) Cognitive impairment: an independent predictor of excess mortality: a cohort study. Ann Intern Med 155:300-308

29. Tas U, Verhagen AP, Bierma-Zeinstra SM et al (2007) Prognostic factors of disability in older people: a systematic review. Br J Gen Pract 57:319-323

30. Pelz I, Pohlabeln H, Reineke A, Ahrens W (2013) Externe Qualitätssicherung der ersten Welle der Studie zur Gesundheit Erwachsener in Deutschland (DEGS1). Bundesgesundheitsbl Gesundheitsforsch Gesundheitsschutz 56:637-642

31. Beauchet O, Fantino B, Allali G et al (2011) Timed Up and Go test and risk of falls in older adults: a systematic review. J Nutr Health Aging 15:933938

32. Guralnik JM, Ferrucci L, Simonsick EM et al (1995) Lower-extremity function in persons over the age of 70 years as a predictor of subsequent disability. N Engl J Med 332:556-561

33. Rivera JA, Fried LP, Weiss CO, Simonsick EM (2008) At the tipping point: predicting severe mobility difficulty in vulnerable older women. J Am Geriatr Soc 56:1417-1423

34. Runge M, Hunter G (2006) Determinants of musculoskeletal frailty and the risk of falls in old age. J Musculoskelet Neuronal Interact 6:167-173

35. Freiberger E, Vreede P de, Schoene D et al (2012) Performance-based physical function in older community-dwelling persons: a systematic review of instruments. Age Ageing 41:712-721

36. Rossiter-Fornoff JE, Wolf SL, Wolfson LI, Buchner DM (1995) A cross-sectional validation study of the FICSIT common data base static balance measures. Frailty and injuries: cooperative studies of intervention techniques. J Gerontol A Biol Sci Med Sci 50:M291-M297

37. Deandrea S, Lucenteforte E, Bravi F et al (2010) Risk factors for falls in community-dwelling older people: a systematic review and meta-analysis. Epidemiology 21:658-668

38. Andersen-Ranberg K, Petersen I, Frederiksen $\mathrm{H}$ et al (2009) Cross-national differences in grip strength among 50+year-old Europeans: results from the SHARE study. Eur J Ageing 6:227-236

39. Bohannon RW (2008) Hand-grip dynamometry predicts future outcomes in aging adults. J Geriatr Phys Ther 31:3-10

40. Cruz-Jentoft AJ, Baeyens JP, Bauer JM et al (2010) Sarcopenia: European consensus on definition and diagnosis: report of the European Working Group on Sarcopenia in Older People. Age Ageing 39:412-423

41. Muir SW, Gopaul K, Montero Odasso MM (2012) The role of cognitive impairment in fall risk among older adults: a systematic review and meta-analysis. Age Ageing 41:299-308

42. Atkinson HH, Rapp SR, Williamson JD et al (2010) The relationship between cognitive function and physical performance in older women: results from the women's health initiative memory study. J Gerontol A Biol Sci Med Sci 65A:300-306

43. Lampert T, Kroll L, Müters S, Stolzenberg H (2013) Messung des sozioökonomischen Status in der Studie zur Gesundheit Erwachsener in Deutschland (DEGS1). Bundesgesundheitsbl Gesundheitsforsch Gesundheitsschutz 56:631-636

44. Semsei I (2000) On the nature of aging. Mech Ageing Dev 117:93-108 


\section{Leitthema}

45. Cesari M, Vellas B, Gambassi G (2012) The stress of aging. Exp Gerontol 48:451-456

46. Kocka J, Staudinger UMH (2009) Gewonnene Jahre. Empfehlungen der Akademiengruppe Altern in Deutschland. Wissenschaftliche Verlagsgesellschaft mbH Stuttgart, Halle (Saale)

47. Bohannon RW (2006) Reference values for the timed up and go test: a descriptive meta-analysis. J Geriatr Phys Ther 29:64-68

48. Rockwood K, Awalt E, Carver D, MacKnight C (2000) Feasibility and measurement properties of the functional reach and the timed up and go tests in the Canadian study of health and aging. J Gerontol A Biol Sci Med Sci 55:M70-M73

49. Whitney SL, Wrisley DM, Marchetti GF et al (2005) Clinical measurement of sit-to-stand performance in people with balance disorders: validity of data for the Five-Times-Sit-to-Stand Test. Phys Ther 85:1034-1045

50. Bohannon RW (2006) Reference values for the Five-Repetition Sit-to-Stand Test: a descriptive meta-analysis of data from elders. Percept Mot Skills 103:215-222
51. Gatenby R, Hayley C (2007) Physical performance. In: Craig R, Mindell J (Hrsg) Health survey for England. General health of older people. The Information Centre, Leeds, S 97-120

52. Rantanen T, Avlund K, Suominen H et al (2002) Muscle strength as a predictor of onset of ADL dependence in people aged 75 years. Aging Clin Exp Res 14:10-15

53. Metter EJ, Talbot LA, Schrager M, Conwit R (2002) Skeletal muscle strength as a predictor of allcause mortality in healthy men. J Gerontol A Biol Sci Med Sci 57:B359-365

54. Elst W van der, Boxtel MP van, Breukelen GJ van, Jolles J (2006) The Letter Digit Substitution Test: normative data for 1,858 healthy participants aged 24-81 from the Maastricht Aging Study (MAAS): influence of age, education, and sex. J Clin Exp Neuropsychol 28:998-1009

\section{Hier steht eine Anzeige.}

\section{Springer}

